\title{
Evolução das Técnicas de Simulação
}

\author{
Elídio de Carvalho Lobão \\ Arthur José Vieira Porto \\ Escola de Engenharia de Säo Carlos - EESC/USP \\ Departamento de Eng. Mecânica
}

Resumo

Este trabalho apresenta a evoluçāo dos sistemas de simulaçāo de acordo com o desenvolvimento tecnológico. Serāo discutidos os simuladores matemáticos, os modelos físicos em escala, as linguagens se simulaçāo de propósito geral e os simuladores dedicados. Finalizando, discutiremos a arquitetura da nova geraçāo de simuladores viabilizada pela evoluçăo das tecnologias eletrônica e de informática: os simuladores interativos e inteligentes, que combinam recursos de realidade virtual e inteligência artificial.

\section{Abstract}

This work describes the simulation's systems evaluation in the light of technology development. Matemathical simulators and reduced physical scale models such as the general purpose simulation language and customized simulators are discussed. Finally, an architeture for the new generation of simulators viabilized by the electronics and informatic state of the art: intelligent and interactive simulators which integrate the artificial intelligence and virtual reality technology is presented.

\section{Palavras Chaves:}

Simuladores, inteligência artificial, sistemas especialistas, realidade virtual.

Keywords:

Simulators, expert systems, virtual reality.

\section{1 - Introdução}

A simulação é uma das mais poderosas ferramentas de análise disponível para projeto e operação de sistemas. A realização de um estudo de simulação antes da implantação do sistema real é muito importante porque permite a aceleração do funcionamento do sistema no tempo, possibilita prever os quase inevitáveis acidentes que ocorrem quando da implantação de um sistema real além de poupar recursos econômicos, pois dispensa a construção de protótipos para testes. A simulação permite ainda, durante o desenvolvimento do projeto de uma nova planta, evitar gargalos, definir o melhor arranjo físico e até determinar o melhor índice de produtividade dos funcionários. A simulação pode ser útil em qualquer uma das fases do ciclo de vida de um sistema de manufatura: desde a fase de análise do problema e definição de requisitos, até as fases de projeto, justificação, implementação e operação.

Através de estudos de simulação pode-se realizar inferências sobre atividades nos sistemas de manufatura tais como: identificação de problemas, da utilização da capacidade instalada, níveis de inventário, lógica de controle, comparação com o desempenho de outros sistemas, refinamento de projeto, integração, alternativas de seqüenciamento, inicialização de equipamentos, treinamento de empregados, etc.

Devido a sua grande versatilidade e flexibilidade, a simulação é largamente utilizada em operações técnicas e atividades de pesquisa. Algumas das muitas áreas de aplicação da simulação são: sistemas de computação, sistemas de manufatura, negócios, 
PRODUÇÃO

entidades governamentais, ecologia e meio - ambiente, sociedade e comportamento, biociências, etc.

As ferramentas de simulação desenvolvidas por engenheiros e projetistas para estudo dos mais diversos sistemas apresentam uma evolução diretamente ligada com a tecnologia de suporte (hardware e software) disponíveis no momento de sua implementação, tendo evoluído desde os modelos físicos em escala e os modelos matemáticos até a última geração de simuladores inteligentes e interativos com interface gráfica. Apresenta-se na tabela I a evolução dos sistemas de simulação em paralelo com o desenvolvimento da tecnologia de suporte, temas os quais serão detalhados no desenrolar deste trabalho.
2- Tipo I: Modelos matemáticos e modelos em escala reduzida

As primeiras ferramentas de modelagem empregadas em simulação foram a modelagem matemática e modelagem em escala, de acordo com a tecnologia disponível na época. Os modelos físicos em escala são reproduções do sistema real cujas características construtivas devem reproduzir em maior ou menor escala geométrica as do modelo real, observando-se as mesmas características físicas e estruturais dos mesmos. Com os dados obtidos a partir de estudos realizados sobre estes modelos pode-se realizar inferências sobre o sistema real.

\begin{tabular}{|c|c|c|}
\hline Classificação & tecnologia disponível & ferramentas de simulação \\
\hline Tipo I & $\begin{array}{l}\text { conhecimentos científicos, } \\
\text { matemáticos, estatísticos e } \\
\text { ferramentas manuais. }\end{array}$ & $\begin{array}{l}\text { modelos matemáticos e modelos físic } \\
\text { em escala reduzida ou ampliada. }\end{array}$ \\
\hline Tipo II & $\begin{array}{l}\text { computadores de grande porte } \\
\text { (main frames), primeiros } \\
\text { microcomputadores. }\end{array}$ & $\begin{array}{l}\text { - linguagens de simulação: gpss, sla } \\
\text { siman, etc. } \\
\text { - simuladores de interface por diálogo. }\end{array}$ \\
\hline Tipo III & microcomputadores. & $\begin{array}{l}\text { Simuladores de interface gráfica - aren } \\
\text { automod, promodel, etc. }\end{array}$ \\
\hline Tipo IV & $\begin{array}{l}\text { estações de trabalho, } \\
\text { microcomputadores de alto } \\
\text { desempenho e grande capacidade } \\
\text { de memória. }\end{array}$ & $\begin{array}{l}\text { Simuladores de interface gráfic } \\
\text { interativos (utilizando recursos } \\
\text { realidade virtual) e inteligent } \\
\text { (utilizando sistemas especialistas } \\
\text { inteligência artificial). }\end{array}$ \\
\hline
\end{tabular}

Tabela T - Evolução dos sistemas de simulação 
Os modelos matemáticos são desenvolvidos a partir de inferências sobre os sistemas dos quais deseja-se obter projeções sobre seu comportamento em determinadas circunstâncias. A partir de observações pode-se então identificar as variáveis que influenciam em seu comportamento e a maneira como elas se interrelacionam, propondo-se então uma relação matemática que descreva o sistema e que permita prever o comportamento do mesmo sob as mais diversas situações. Algumas aplicações práticas para os modelos matemáticos podem ser citados (Braun, 1979): para verificação da autenticidade de obras de arte, estudos da variação populacional (modelo de Malthus), estudo da dinâmica de crescimento de um tumor, estudos sobre vibrações mecânicas, estudo do diabetes, inferências sobre campanhas militares com os modelos de F. W. Lanchester, etc.

\section{1 - Situação atual}

Com o desenvolvimento da eletrônica e da informática, aos modelos físicos em escala foram incorporados recursos tais como microprocessadores e micro-sensores eletrônicos que aumentaram em muito a capacidade de obtenção de dados dos mesmos. Atualmente, alguns kits microprocessados destes modelos possuem capacidade de movimentação e permitem por exemplo, a simulação de linhas de manufatura (Ranky, 1983). O acoplamento de sensores eletrônicos de alto desempenho aos mesmos permite também por exemplo, o estudo de aerodinâmica e de esforços estruturais. Uma outra técnica para construção de modelos físicos em escala é descrita por Schlie e Grimberg (Schlie e Grimberg, 1995), que relatam a construção de modelos físicos utilizando-se da técnica de estereolitografia. Esta técnica consiste de um feixe de laser ultravioleta que incide sobre um recipiente que contém uma resina foto-sensível, que solidifica-se ao contato com a luzU. O movimento deste feixe de laser é controlado por um computador de acordo com o projeto da peça que se deseja construir.

No entanto, a técnica de modelagem física em escala possui algumas desvantagens: custo elevado (Ramos, 1995; Daimler-Benz, 1995 - I), complexidade de seu processo construtivo, e inflexibilidade de readaptação para outros sistemas (Elmariaghi e Ravi, 1989).

Já os modelos matemáticos receberam um enorme impulso com o desenvolvimento da tecnologia de informática, e hoje há diversos pacotes comerciais de software que permitem simular modelos dos mais complexos sistemas, tais como: o MATLAB, o MATRIX, SIMULINK, etc. Estes softwares permitem simular e realizar inferências também sobre modelos construídos em outros pacotes simuladores, tais como: ADAMS e SDFAST para sistemas multi-corpos, CAEDS, ANSYS, NASTRAM E COSMOS para estudos de elementos finitos, etc.

\section{3 - Tipo II: linguagens de simulação}

O acirramento da competitividade em decorrência do processo de globalização da economia trouxe para as empresas o desafio do aumento da eficiência de seu sistema produtivo. Isto implicou na modernização das plantas industriais, reposição de equipamentos obsoletos e alteração de "layouts", com uso intensivo de sistemas automatizados. Estes sistemas, que possibilitam altos ganhos de produtividade (devido ao menor desperdício de tempo para inicialização dos equipamentos e maior eficiência no processo), qualidade (pela uniformização do produto) e rentabilidade (com índices menores de desperdício e maiores de produção) são cada vez mais complexos, e seu monitoramento e operação por parte de operadores humanos torna-se arriscado devido à complexidade e quantidade das ações 
corretivas a serem desencadeadas em caso de pane. Isto tudo implica em custos cada vez mais altos de inicialização de sistemas, de construção de protótipos, sistemas de treinamento, etc.;

Esta situação trouxe consigo uma demanda por sistemas de simulação, os quais permitem que estas tarefas (como projetos ou "up-grades" desistemas complexos, inicialização dos mesmos e treinamento dos operadores) possam ser realizados em tempo mais curto, sem riscos para os equipamentos e operadores e sem necessidade de parada da produção. Inicialmente, os sistemas de simulação foram desenvolvidos sobre linguagens de programação de propósito geral (Basic, Pascal, C, etc.). No entanto, isto demandava um grande esforço para construção de modelos, além de exigir do profissional responsável pela simulação conhecimentos profundos de programação de computadores. Foi então que apareceram as linguagens de programação de computadores dedicadas á simulação, tais como GPSS, SIMAN, SLAM, SIMSCRIPT, etc. Estas linguagens na realidade eram bibliotecas compostas de conjuntos de macro-comandos de outras linguagens de propósito geral (na maioria das vezes, FORTRAN), e conforme já mencionado anteriormente; visavam facilitar o processo de construção dos modelos. Alguns dos simuladores gráficos da geração seguinte são desenvolvidos sobre plataforma destas linguagens, como no caso do ARENA, construído sobre a linguagem SIMAN (ARENA, 1994).

4 - Tipo III: simuladores de interface gráfica e orientados à objeto.

$\mathrm{O}$ aparecimento das linguagens específicas para desenvolvimento de modelos de simulação aliado a diminuição dos custos de hardware e software contribuiu para o aumente do número de usuários $\mathrm{e}$ de aplicações da técnica de simulação de sistemas.
Este maior número de usuários tornou viável o desenvolvimento de programas simuladores específicos para determinados sistemas: simuladores de manufatura (Strandhagen, 1987), simuladores de vôo (Murillo e Arantes, 1995), circuitos eletrônicos, simuladores de trafego (OESP, 1995), treinamento para condutores de trens (Daimler-Benz, 1995 II), etc. Os simuladores são pacotes de software projetados para facilitar a modelagem de sistemas em determinados ambientes, geralmente tendo então seu escopo de aplicação reduzido á estes domínios (um simulador de vôo por exemplo, não pode ser utilizado para simular um sistema de manufatura). Embora os simuladores não apresentem a mesma flexibilidade das linguagens de simulação de propósito geral, apresentam algumas vantagens em relação a elas:

- interação usuário/computador mais amigável;

- possibilidade de utilização de biblioteca de rotinas já previamente construídas, e

- os simuladores dispensam o usuário da necessidade de ter conhecimentos profundos de programação de computadores, pois situam-se num nivel mais alto de programação.

Embora os primeiros simuladores ainda não apresentassem uma interface muito amigável com o usuário, com o desenvolvimento tecnológico e aumento do poder de processamento dos computadores pessoais (aumento da velocidade de processamento, da capacidade de memória e facilidade de desenvolvimento de sistemas de interface gráfica sobre o Windows), vários sistemas simuladores comerciais foram colocados no mercado, tais como por exemplo: ARENA, PROMODEL, AUTOMOD, etc. Estes sistemas apresentam como características:

- interface gráfica de comunicação com usuário;

- desenvolvimento de modelos orientados a objeto; 
- capacidade de animação do modelo;

- fornecimento de relatórios sobre a simulação executada;

- ferramentas estatísticas para tratamento dos dados utilizados na simulação, etc.

5 - Tipo IV: simuladores interativos e inteligentes.

A nova tendência que se apresenta para os sistemas simuladores aponta para sistemas interativos e inteligentes, nos quais serão largamente empregados recursos de realidade virtual, inteligência artificial e sistemas especialistas, de acordo com a figura 1 a seguir:
Os novos sistemas simuladores tendem a tornar-se um "Shell" composto por vários módulos que interagem entre si, e auxiliam no processo de simulação desde a determinação das especificações do processo e do modelo a ser construído até a obtenção dos dados finais e sua posterior análise. A seguir, são apresentados comentários breves sobre cada um dos diversos módulos do simulador e a interação entre eles e os usuários do sistema.

5.1 - Sistema especialista: uma das principais funções de um sistema especialista é transferir os conhecimentos que poucos especialistas possuem sobre determinado assunto a um vasto grupo de

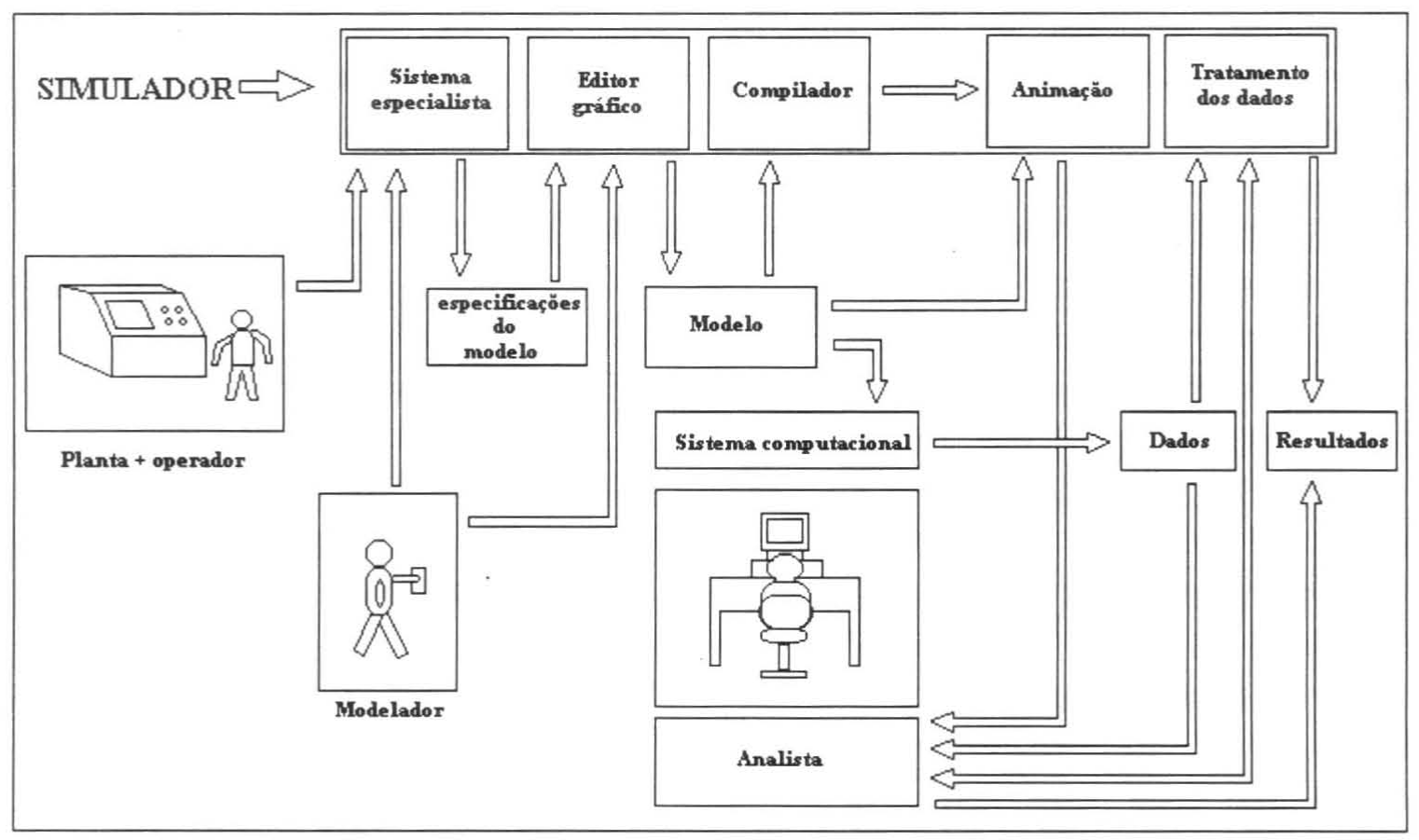

Figura 1 - sistemas simuladores interativos e inteligentes 
PRODUÇÃO

usuários. Os simuladores de nova geração deverão contar com um sistema especialista para auxiliar no processo de modelagem, pois os resultados de um processo de simulação são totalmente relacionados com a maneira como o modelo representa o modelo real - modelos mal formulados fatalmente produzirão resultados que não descrevem de maneira adequada a realidade (Lobão e Porto, 1995). Neste caso, o profissional responsável pelo desenvolvimento do modelo, de posse dos dados obtidos do monitoramento da operação do sistema pode então, com auxílio do sistema especialista estabelecer os dados de entrada necessários e definir os experimentos de simulação a serem realizados. Wichmann (Wichmann, 1987) sugere a classificação dos sistemas especialistas em quatro categorias, quando estes atuam em conjunto com um sistema de simulação:

- Sistema especialista como sistema consultivo separado: um sistema especialista não totalmente integrado com o sistema de simulação, atua como um "manual de usuário inteligente".

- Sistema especialista integrado e interfaceado com uma linguagem convencional de simulação: atua como um "front-end" para preparação do modelo, especificação dos dados de entrada e definição dos experimentos de simulação. Pode conter informações do tipo como interpretar resultados, quais as medidas são apropriadas, quantas replicações são necessárias, etc.

- Sistema especialista integrado e interfaceado com um sistema simulador existente: contém conhecimentos dentro do escopo do simulador com o qual está integrado. Geralmente necessita de conhecimentos do tipo "estrutural" (sobre o simulador), "estratégico" (sobre o processo simulado) e "de raciocínio" (características de Inteligência Artificial)

- Linguagem de simulação baseada no conhecimento: um passo adiante das metodologias convencionais de simulação. Apresenta estruturas separadas para base de dados, base de conhecimento, e estrutura de controle; permitindo que cada um destes módulos possa ser modificado sem afetar os outros.

\section{2 - Editor gráfico: o editor gráfico é um} módulo de interface entre o usuário e a linguagem de baixo nível (muitas vezes uma linguagem de simulação, como por exemplo o SIMAN), que permite que a construção do modelo seja executada de uma forma orientada a objeto - dispensando desta forma o usuário da necessidade de possuir conhecimentos mais profundos em programação de computadores. Strandhagen (Strandhagen, 1987) ressalta que a abordagem de orientação a objeto permite também otimizar o processo de construção dos modelos, pois os mesmos podem ser construídos como uma coleção de objetos, da forma como são vistos na vida real. Estes objetos atuam como caixas pretas parametrizadas onde o usuário tem apenas que fornecer os seus parâmetros. A figura 2 mostra a edição de objetos no software de simulação PROMODEL.

\section{3 - Compilador: o compilador é o} interpretador do módulo gráfico, onde foi construído o modelo orientado a objeto. O compilador é geralmente pode ser uma linguagem de mais baixo nível, tal como SIMAN, GPSS, SLAM (linguagens de simulação), ou C, Pascal, Fortran (linguagens de propósito geral), etc.

\section{4 - Módulo de animação: é a parte do} software que realiza a "linkagem" entre o editor gráfico e compilador com o usuário, "mostrando" ao mesmo a interação entre os diversos componentes do modelo durante a execução de uma corrida de simulação. Alguns módulos animadores conhecidos são por exemplo, o CINEMA e o PROOF ANIMATOR. A tendência para a nova geração de simuladores é a adoção de ambientes de realidade virtual para realização desta tarefa, o que permitirá 


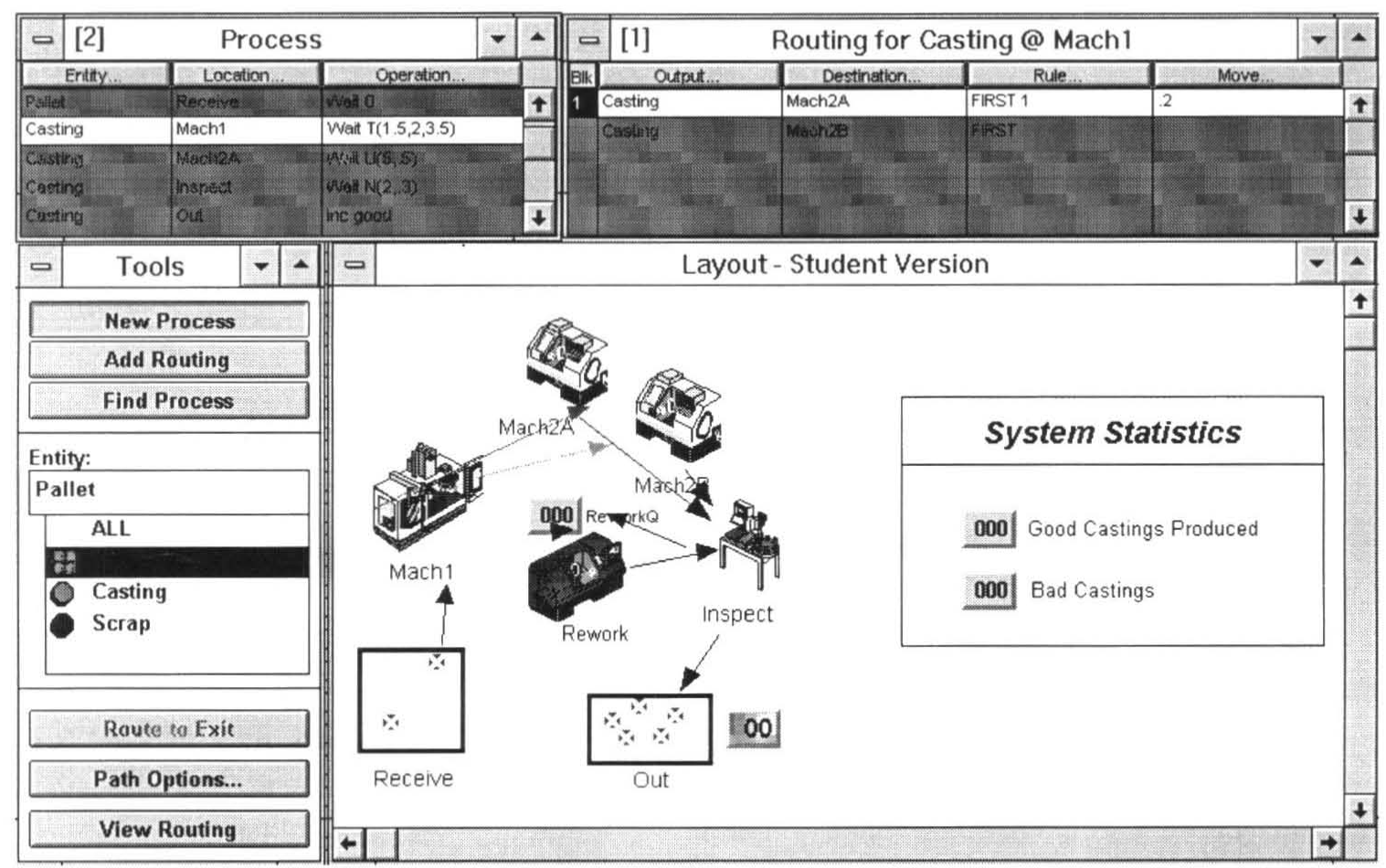

Figura 2 - programação orientada a objeto no PROMODEL

ao usuário não somente interagir com os componentes do sistema durante seu "funcionamento virtual" bem como muitas vezes, "imergir" no interior do modelo - dando mais realismo ao sistema e permitindo uma exploração mais rica do mesmo.

\section{5 - Módulo de tratamento dos dados: o} módulo de tratamento dos dados objetiva auxiliar o analista na interpretação dos dados resultantes da simulação do modelo, desobrigando-o da necessidade de possuir profundos conhecimentos estatísticos. Este módulo também pode contar com recursos de inteligência artificial e uma base de conhecimento de forma a permitir inferências sobre os resultados obtidos.

\section{6 - Conclusões}

O desenvolvimento das ferramentas de simulação, sob certos aspectos pode ser visto como uma conseqüência da globalização econômica e o decorrente aumento da competitividade; e foi bastante favorecido pela veloz evolução tecnológica da eletrônica e da informática. O aumento da competitividade exige das empresas cortes substanciais tanto nos custos de desenvolvimento de novos produtos quanto de operação das plantas e treinamento de recursos humanos, e a simulação é uma ferramenta que possibilita a realização de tais tarefas efetivamente reduzindo custos quando estes são comparados com os custos da realização das mesmas utilizando sistemas físicos reais. A evolução tecnológica vivenciada permite que o aumento do poder de análise de tais sistemas simuladores ocorra paralelamente com a diminuição dos preços dos 
mesmos, realimentando positivamente o processo e criando um ciclo virtuoso que trás incentivos tantos para os usuários quanto para os desenvolvedores destes sistemas simuladores.

Os primeiros sistemas de simulação que surgiram exigiam dos profissionais que trabalhavam nesta área conhecimentos sobre o processo a ser simulado, programação de computadores e ferramentas estatísticas para interpretação dos resultados apresentados. A nova geração de sistemas simuladores que tende a ocupar o mercado apresenta uma série de evoluções que facilitarão o processo de modelagem e simulação:

- construção de modelos com orientação ao objeto permitem que os mesmos sejam construídos como uma coleção de objetos que se interrelacionam. A abordagem orientada a objeto permite que o usuário construa modelos da forma com que vê o sistema na vida real: como uma coleção de objetos. Isto contorna a necessidade de o usuário precisar de conhecimentos em programação de computadores;

- o emprego de técnicas de realidade virtual poderá permitir que o usuário interaja com o sistema durante a simulação, enriquecendo desta forma a qualidade dos dados obtidos e muitas vezes evitando a necessidade da realização de várias corridas para se chegar a uma quantidade necessária dẹ dados para realização de inferências estatísticas;

- módulos de sistemas especialistas auxiliarão a equipe de simulação na fase de definição das especificações do sistema a definir as principais diretrizes para construção do modelo, auxiliando desta forma profissionais que não tenham profundos conhecimentos sobre o processo;

- na análise e interpretação dos resultados obtidos também o analista poderá contar com auxílio de módulos inteligentes para interpretação dos resultados.

\section{7-Bibliografia}

(Arena, 1994): ARENA SIMAN reference guide. Systems Modeling Corporation, Sewickley, PA, USA, 1994. 451 p.

(Braun, 1979): Braun, Martin: "Equações diferenciais e suas aplicações". Editora Campus. Rio de Janeiro, 1979. 378 p.

(Daimler-Benz, 1995-I): Daimler-Benz cover story: "Test dive the E-class." Daimler-Benz HighTech Reports, n3, 1995 pp 40 - 49.

(Daimler-Benz, 1995-II): Daimler-Benz Mosaic: "Rolling down Virtual Rails." Daimler-Benz HighTech Reports, n3, 1995 pp 68-69.

(ElMariaghy e Ravi, 1995): ElMariaghy, H. A.; Ravi, T.: "Novas ferramentas para projeto, modelamento e avaliação de FMS." Máquinas e Metais, Abril 1995 pp 28 - 41.

(Lobão e Porto, 1995): Lobão, E. C.; Porto, A. J. V.: "Uma técnica de modelagem para simulação de máquinas que leva a um bom produto." Máquinas e Metais, Dezembro de 1995 pp 22 - 34.

(Murillo e Arantes, 1995): Murillo, Carlos; Arantes, Fábio G.: "Simulador de vôo desafia pilotos de acrobacia", Jornal "Folha de S. Paulo", 6 de setembro de 1995, pag 6-12.

(OESP, 1995): O Estado de São Paulo. "Peritos usam simulador para desvendar acidente de transito" Jornal "O Estado de São Paulo", 11 de dezembro de 1995, pag G-31. 
(Ramos, 1995): Ramos, T. O.: "Maquete eletrônica ajuda a fazer jato" Jornal "O Estado de São Paulo", 27 de novembro de 1995, pag G-16.

(Ranky, 1983): Ranky, Paul: "The design and operation of FMS". North Holland Publishing Company. - U.K. 1983, 343 p.

(Schlie e Grimberg, 1995): Schlie, P. G.; Grimberg, P. E.: "Estereolitografia, una hierramenta poderosa". Jornadas internacionales de mecanica computacional y $\mathrm{Cad} / \mathrm{Cam}$. Anais do congresso, pp 449-455, Concepción, Chile. Novembro de 1995.

(Strandhagen, 1987): Strandhagen, J. O.: "Expert knowledge in object-oriented simulation of manufacturing systems" Proceedings of 3rd international conference of simulation in manufacturing. Turin, Italy, nov. 1987 pp $23-30$.

(Wichmann, 1987): Wichmann, K.E.: "Trends in the development of simulation software tools for analyzing manufacturing systems" Proceedings of 3rd international conference of simulation in manufacturing. Turin, Italy, nov. 1987 pp 39 - 48. 